\title{
XLVI. A reciprocal relation in diffraction
}

\section{A.A. Michelson}

To cite this article: A.A. Michelson (1905) XLVI. A reciprocal relation in diffraction, Philosophical Magazine Series 6, 9:52, 506-507, DOI: 10.1080/14786440509463300

To link to this article: http://dx.doi.org/10.1080/14786440509463300

册 Published online: 08 Jun 2010.

Submit your article to this journal $\pi$

LII Article views: 1

Q View related articles $₫$

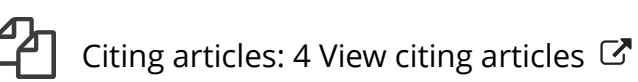




\section{$506]$ \\ XLVI. A Reciprocal Relation in Diffraction.}

By A. A. Michelson *.

SUPPOSE the vibration at the surface $A$ of a sphere whose centre is at $O$ to be a known function of $x$ and $y$, the origin being a point $P$ on the sphere,

$$
\mathrm{V}=\phi \cos n t+\psi \sin n t
$$

Then the vibration $\dagger$ on a sphere $B$ passing through $O$ whose centre is at $P$ will be

$$
\mathrm{W}=-\frac{1}{\lambda f} \iint \phi d x d y \sin n(t-\tau)-\frac{1}{\lambda f} \iint \psi d x d y \cos n(t-\tau)
$$

Putting

$$
\begin{gathered}
\tau=\frac{f}{a}\left(1-\frac{x \xi+y \eta}{f^{2}}\right), \quad t-\frac{f}{a}=t_{1}, \quad \frac{n \xi}{f a}=u, \quad \frac{n \eta}{f a}=v, \\
\mathrm{~W}=-\frac{1}{\lambda f}\left[\iint \phi d x d y \cos (u x+v y)-\iint \psi d x d y \sin (u x+v y)\right] \sin n t \\
-\frac{1}{\lambda f}\left[\iint \phi d x d y \sin (u x+v y)+\iint \psi d x d y \cos (u x+v y)\right] \cos n t
\end{gathered}
$$

If now a spherical mirror be made to coincide with the sphere $B$ an image of the source will be formed at $A$.

This image may also be considered as the resultant of the vibrations at $B$. Hence, if we designate by DW the

* Communicated by the Author.

† Scientific Papers of Lord Rayleigh, vol. iii. p. 80. The results given by Lord Rayleigh for the intensity of the diffraction figure in the focal plane do not apply to the phase of the vibration. This restriction is removed if the surface considered be the sphere $B$; for the distance between two points on the spheres is

$$
\begin{aligned}
\rho^{2} & =(x-\xi)^{2}+(y-\eta)^{2}+(z-\zeta)^{2}, \\
\text { or if } \quad f^{2}=x^{2}+y^{2}+z^{2} & =\xi^{2}+\eta^{2}+(\zeta-f)^{2} \\
\rho^{2} & =f^{2}-2 x \xi-2 y \eta-2 z \zeta+2 f \zeta .
\end{aligned}
$$

If $\xi$ and $\eta$ are small, $\zeta$ will be of the second order, and so is $f-z$, so that $(z-f) \zeta$ is of the fourth order and may be neglected. Hence

or approximately

$$
\begin{aligned}
\rho^{2} & =f^{2}-2 x \xi-2 y \eta \\
\rho & =f-\frac{x \xi+y \eta}{f} .
\end{aligned}
$$


operation by which $\mathrm{W}$ is obtained from $\mathrm{V}$,

$$
\begin{aligned}
& \left.\begin{array}{l}
\mathrm{W}=\mathrm{DV} \\
\mathrm{V}=\mathrm{DW}
\end{array}\right\} \cdot \cdot \cdot \cdot \cdot \cdot \\
& \mathrm{V}=\mathrm{DDV} \text {. }
\end{aligned}
$$

These equations hold for light of any colour, and-with corresponding extension of the definition of $\mathrm{D}$-to any combination of colours.

The analogy with Fourier's formula is apparent. In fact, the second of equations (5) is, putting $n / f a=p$,

$$
\begin{aligned}
\mathrm{V}= & -\frac{1}{\lambda f}\left[\iint \mathrm{Q} d \xi d \eta \cos p\left(x_{1} \xi+y_{1} \eta\right)-\iint \mathrm{P} d \xi d \eta \sin p\left(x_{1} \xi+y_{1} \eta\right)\right] \cos n t_{2} \\
& -\frac{1}{\lambda f}\left[\iint \mathrm{Q} d \xi d \eta \sin p\left(x_{1} \xi+y_{1} \eta\right)+\iint \mathrm{P} d \xi d \eta \cos p\left(x_{1} \xi+y_{1} \eta\right)\right] \sin n t_{2}
\end{aligned}
$$

Substituting the values of $P$ and $Q$ from (4),

$$
\left.\begin{array}{rl}
\mathrm{V} & =\frac{1}{\lambda^{2} f^{2}} \iiint \int_{\int} d x d y d \xi d \eta \psi(x, y) \sin \left[\left(x_{1}-x\right) p \xi+\left(y_{1}-y\right) p \eta\right] \\
& +\frac{1}{\lambda^{2} f^{2}} \iiint d x d y d \xi d \eta \phi(x, y) \cos \left[\left(x_{1}-x\right) p \xi+\left(y_{1}-y\right) p \eta\right]
\end{array}\right\} \cos n t_{2}
$$

The first and third integrals are identically zero, so that putting

$$
u=\frac{n \xi}{f a} \quad \text { and } \quad v=\frac{n \eta}{f a}
$$

and disregarding the phase difference between $t$ and $t_{2}$, we have by (1)

$\frac{4 \pi^{2}}{f^{2}} \phi\left(x_{1}, y_{1}\right)=\iiint \int d u d v d x d y \phi(x, y) \cos \left[\left(x_{1}-x\right) u+\left(y_{1}-y\right) v\right]$

This, disregarding the intensity factor $f^{\mathfrak{Z}}$, is the Fourier formula extended to two dimensions.

Formulæ (5) express the fact that if

$\mathrm{W}$ is the diffraction image of $\mathrm{V}$, then

$V$ is the diffraction image of $W$.

In applying the formulæ it must be remembered that $V$ and $W$ represent the vibration-not merely the intensity. 